\title{
First records of Lasiodiamesa gracilis (Kieffer, 1924), Parochlus kiefferi (Garrett, 1925) and several other Chironomidae from the Czech Republic and Slovakia
}

\author{
Vít Syrovátka ${ }^{1}$ and Peter H. Langton ${ }^{2}$ \\ ${ }^{1}$ Department of Botany and Zoology, Masaryk University, Kotlářská 2, Brno CZ-611 37, Czech Republic. \\ E-mail: syrovat@sci.muni.cz \\ ${ }^{2}$ University Museum of Zoology, Cambridge, Downing Street, Cambridge UK (address for corresponden- \\ ce: 16, Irish Society Court, Coleraine, BT52 1GX Northern Ireland, United Kingdom). \\ E-mail: langtonph@gmail.com
}

The species composition of the Chironomidae fauna in the Czech Republic is still poorly known despite a long tradition in Chironomidae research, already established by Prof. Jan Zavřel in the beginning of the $20^{\text {th }}$ century. The major reasons are a low number of researchers dealing with Chironomidae in the Czech Republic and their traditional focus on larvae, which usually cannot be identified to species. As a result, only a small portion of the chironomid fauna living in the Czech Republic has been discovered so far and many chironomid species are first to be recorded in the future. This concerns both (common) species that are already known from Slovakia and other neighbouring countries, and species living in poorly studied habitats, such as montane springs or bogs.

Times are getting better. Recently, Ivan Skála (2011, 2012, 2013, 2014) contributed to the list of species known from the Czech Republic and the last Chironomidae Symposium revealed even a new subfamily (Ashe et al. 2014).

The first author of this paper came across some new records as well, and this is the first time an annotated list of these species from the Czech Republic is provided.

\section{Podonominae}

In Europe, the representatives of the subfamily Podonominae are found mostly in Scandinavia, but they are also known from several other countries across Europe including all countries neighbouring the Czech Republic (Bitušík and Brabec 2009, Sæther and Spies 2015). The Czech Republic has been an exception. Two species of Podonominae are now recorded from the Czech Republic for the first time.

Lasiodiamesa gracilis (Kieffer 1924)

During the first-author's (VS) visits to his grandmother living in Škrdlovice he regularly collected pupal exuviae from a peat pool within a mineral poor Sphagnum fen, Radostínské rašeliniště National Nature Reserve (Fig. 1). This small reserve is located in the Žd'árské Vrchy Protected Landscape Area within the Bohemian-Moravian Highlands. VS found a single pupal exuviae of Lasiodiamesa on 14 May 2011 and identified it as L. gracilis. Despite the fact that the identification was confirmed by P. Langton at the Chironomid Symposium in Trondheim a few months later, VS felt uncertainty about it because of subtle characters that discriminate this species from L. sphagnicola (Kieffer 1925).

During one of his next visits, 15 March 2015 VS succeeded to collect tens of larvae of Lasiodiamesa from the same locality and reared two male and several female adults. Adult males bear much better characters than pupae and the two males could be easily identified as L. gracilis using the key by Brundin (1966).

The habitat where the species was found corresponds well with the known restriction of the genus to Sphagnum bogs in the Middle Europe, where it is considered a glacial relict, as stated by Brundin (1966). Radostínské rašeliniště Sphagnum bog is the only place in the Bohemian-Moravian Highlands where pools are developed, while the closest Sphagnum bogs with pools may be found as far away as in Sudetenland. Indeed, the closest known locality is the type locality just across the Czech/Poland border in Poland some 90 km to the north from the Radostínské rašeliniště (Brundin 1966, Sæther and Spies 2015). Other populations may be expected in Sudetenland, however VS has been unsuccessful there so far. This might be caused by the rarity of the species as stated by Verberk et al. (2003), but also by unsuitable time of the author's sampling - in late spring and summer the species might be already emerged and can not be found in water anymore. Other known localities in Europe are in The Netherlands (Verberk et al. 2003) and in Scandinavia (Brundin 1966, Sæther and Spies 2015). 


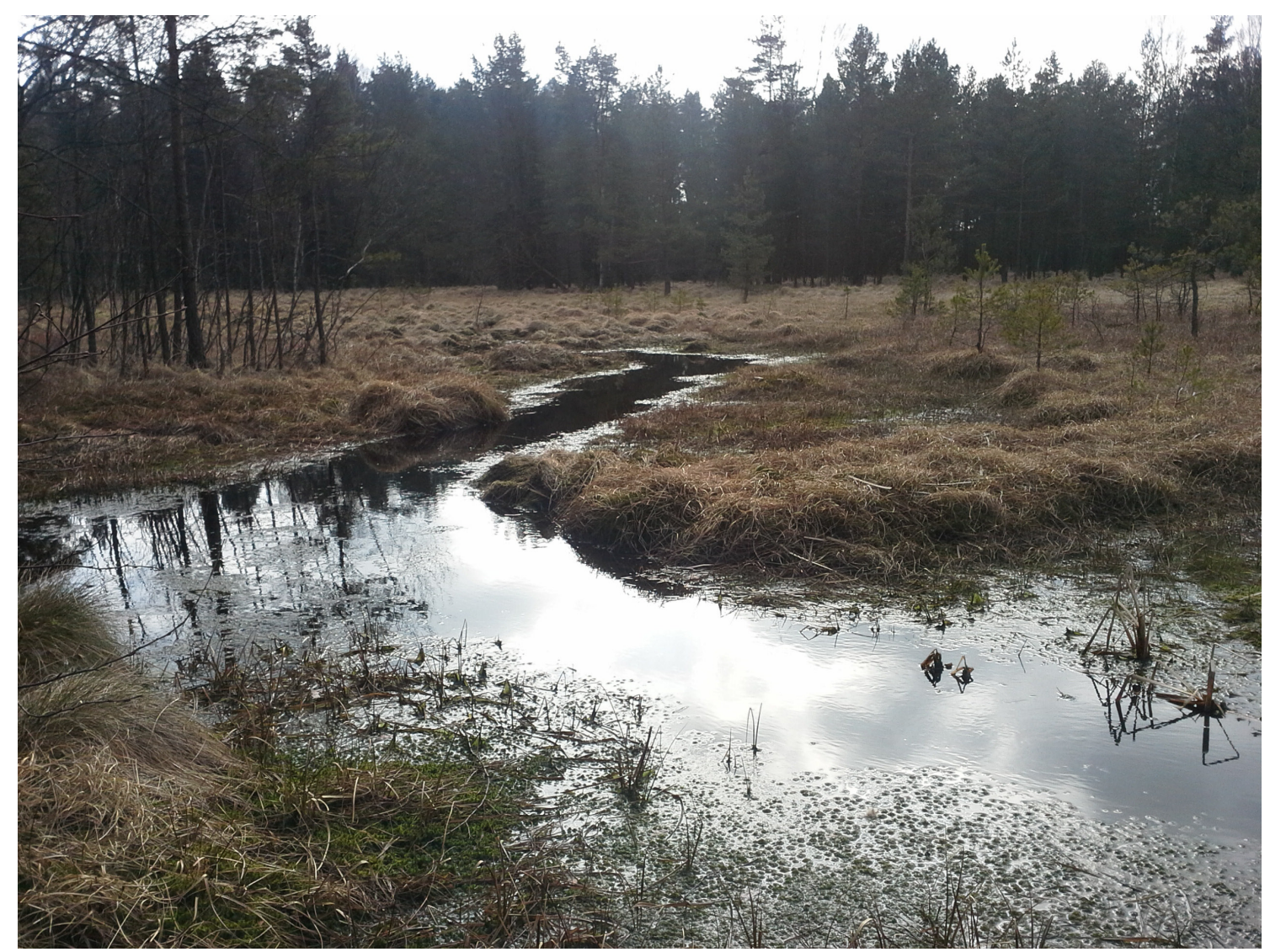

Figure 1. Radostínské rašeliniště National Nature Reserve, Lasiodiamesa gracilis sampling site (site nr. S15 in the List). Photograph taken during sampling 22 March 2015.

The chironomid assemblage at Radostínské rašeliniště was dominated by Monopelopia tenuicalcar (Kieffer 1918) and Corynoneura spp. in early spring, in late spring also by Psectrocladius bisetus Goetghebuer 1942 and Psectrocladius oligosetus Wülker 1956. Throughout the year Macropelopia adaucta Kieffer 1916, Procladius choreus (Meigen 1804), Ablabesmyia phatta (Egger 1864) and Telmatopelopia nemorum (Goetghebuer 1921) were found. Full record information is summarized in Table 1.

\section{Parochlus kiefferi (Garrett 1925)}

On a cross-country skiing trip on 20 February 2015 in the Krkonoše Mountains National Park (Giant Mts.) VS collected a tuft of decomposing vegetation with mosses from a helocrene spring located just next to a ski-track (Fig. 2). This spring is usually covered with snow in the winter, but due to the combination of lack of snowfall during the previous days, sunny weather and slope exposition to the south, it was uncommonly snow-free at that time. The inspection of the collected material back in the laboratory revealed one larva of Parochlus.

The identification of Parochlus larvae into species is not possible, but only a single species $P$. kiefferi is known from the northern hemisphere. Thus, the collected specimen most certainly belongs to this species.

P. kiefferi seems to be cold-stenothermic, living mostly in cold springs and upper courses of spring-fed streams (Brundin 1966). It was also reported from large streams with strong current in North America (Brundin 1966) or from moss in a pond fed by ice-melt water in Italian Alps (Lencioni et al. 2007).

It is possible that the species emerges just after the snow melts and is no longer present at the locality, as was observed by Wülker (1958) in Feldberg springs. Such phenology would prevent the species from being recorded by conventional sampling, which is usually carried out in the vegetation season. It is worth noting, however, that in some regions the species emerges practically throughout the whole snow-free period: from the beginning of June to the beginning of October in Swedish mountains or from the end of April to the beginning of November in temperate Canada (Brundin 1966). In the Italian Alps, Lencioni et al. (2007) recorded a pupal exuviae in the middle of September. Please see Table 1 for summarized information. 


\section{Other species new to the Czech Republic}

Traditionally, the area of the Czech Republic is divided into Bohemia (the western part) and Moravia (the eastern part). New records are therefore designated for these parts separately. Apart from the two Podonominae species we recorded further 111 chironomid species that are new to either part of Czech Republic, to Czech Republic as a whole, or exceptionally to Slovakia. All these records are summarized in Table 1.

As the species were recorded from 65 localities (Appendix 1), we use abbreviations to refer to them in the table. Most localities may be classified either as helocrene springs (abbreviation starts with $H$ followed by a number), flowing waters $(F)$ or standing waters $(S)$. Two highly artificial localities were kept separately as other $(O)$. The details about the localities are given in appendix 1.

\section{Acknowledgements}

We express our gratitude to all who contributed by collecting the material, namely Denisa Němejcová, Petr Komzák, Stanislav Větríček, Michal Straka and Jan Sychra. Our thanks go also to the Czech Science Foundation (P505/11/0779) for financial support of our research in helocrene springs.

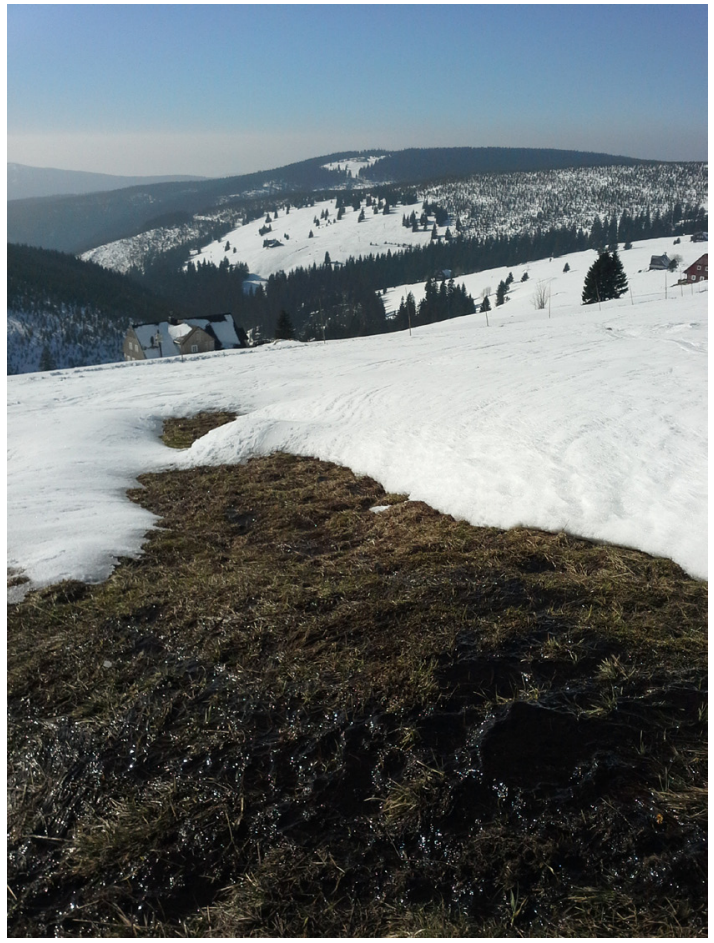

Figure 2. A spring close to Nová Klínovka chalet (in the background) in the eastern part of a meadow called Klínové Boudy, The Krkonoše Mountains National Park. Parochlus kiefferi sampling site (site nr. H11 in the List). Photographed during sampling 20 February 2015.

Table 1. The list of species new to Bohemia (B), Moravia (M), Czech Republic (CZ) or Slovakia (SK). Subfamilies/ tribes are listed taxonomically, species within subfamilies/tribes are in alphabetical order. The column New to describes the area for which a species is reported for the first time. Taxa new to Bohemia or Moravia indicated with B or M have already been reported from the other part of the Czech Republic. Species new to the Czech Republic are indicated with region in parentheses. The column Record details includes date of collection, locality abbreviation (in italic): number of larvae (L), pupae (P), pupal exuviae (PE), imagines (I). All material was identifed by the first author, except where noted. Localities are listed in the following habitat order: Helocrene spring $(\mathrm{H})$, flowing water $(\mathrm{F})$, standing water $(\mathrm{S})$, other $(\mathrm{O})$. See appendix 1 for list of localities.

\begin{tabular}{|c|c|c|}
\hline Species & New to & Record details \\
\hline \multicolumn{3}{|l|}{ PODONOMINAE } \\
\hline Lasiodiamesa gracilis & $\mathrm{CZ}(\mathrm{B})$ & $\begin{array}{l}\text { B: } 14 \text { May 2011, S15: 1PE, det. P. Langton; } 15 \text { Mar } \\
\text { 2015, S15: } 2 \text { reared I }{ }^{\pi}\end{array}$ \\
\hline Parochlus kiefferi & $\mathrm{CZ}(\mathrm{B})$ & B: 22 Feb 2015, H11: 1L \\
\hline \multicolumn{3}{|l|}{ TANYPODINAE-Procladiini } \\
\hline Procladius crassinervis & $\mathrm{CZ}(\mathrm{B})$ & $\begin{array}{l}\text { B: } 29 \text { May 2015, S1: 5PE; } 17 \text { Oct 2013, S7: 1PE, leg. } \\
\text { D. Němejcová. }\end{array}$ \\
\hline \multicolumn{3}{|c|}{ TANYPODINAE-Pentaneurini } \\
\hline Ablabesmyia longistyla & $\mathrm{CZ}(\mathrm{B}, \mathrm{M})$ & $\begin{array}{l}\text { B: } 24 \text { Apr 2007, F28:C6428: 1PE, leg. M. Straka, det. } \\
\text { P. Langton. } \\
\text { M: } 26 \text { Sep 2006, F1: 2PE, det. P. Langton. }\end{array}$ \\
\hline Ablabesmyia phatta & $\mathrm{CZ}(\mathrm{B})$ & $\begin{array}{l}\text { B: } 16 \text { May 2007, S4: 4PE+2L; } 20 \text { May 2007, S15: } \\
\text { 1PE. }\end{array}$ \\
\hline Conchapelopia hittmairorum & $\mathrm{CZ}(\mathrm{M})$ & $\begin{array}{l}\text { M: } 15 \text { Sep 2006, F1: 2PE; } 26 \text { Sep 2006, F1: 1PE, all } \\
\text { det. P. Langton. }\end{array}$ \\
\hline Conchapelopia viator & M & M: 15 Sep 2006, F1: 1PE, det. P. Langton. \\
\hline
\end{tabular}




\begin{tabular}{|c|c|c|}
\hline Species & New to & Record details \\
\hline Hayesomyia tripunctata & $\mathrm{M}$ & M: 23 Aug 2005, F1: 1PE, det. P. Langton. \\
\hline Thienemannimyia pseudocarnea & $\mathrm{B}$ & B: 24 Apr 2007, F28: 4PE, leg. M. Straka. \\
\hline Zavrelimyia barbatipes & $\mathrm{B}$ & B: 21 Aug 2006, F21: 1PE, det. P. Langton. \\
\hline \multicolumn{3}{|l|}{ DIAMESINAE-Tribus Diamesini } \\
\hline Diamesa permacra & $\mathrm{CZ}(\mathrm{M})$ & $\begin{array}{l}\text { M: } 25 \text { Sep 2006, H7: 1PE, leg. J. Bojková, det. P. } \\
\text { Langton. }\end{array}$ \\
\hline Potthastia longimana & $\mathrm{B}$ & B: 24 Apr 2007, F28: 2PE, leg. M. Straka. \\
\hline \multicolumn{3}{|l|}{ PRODIAMESINAE } \\
\hline Odontomesa fulva & $\mathrm{B}$ & B: 24 Apr 2007, F28: 1PE, leg. M. Straka. \\
\hline \multicolumn{3}{|l|}{ ORTHOCLADIINAE } \\
\hline Chaetocladius melaleucus & $\mathrm{CZ}(\mathrm{B}, \mathrm{M})$ & $\begin{array}{l}\text { B: } 24 \text { Feb 2008, F22: 3PE, det. P. Langton. } \\
\text { M: } 25 \text { Sep 2006, H5: 1PE, leg. J. Bojková. }\end{array}$ \\
\hline Chaetocladius perennis & $\mathrm{CZ}(\mathrm{B}, \mathrm{M})$ & $\begin{array}{l}\text { B: } 15 \text { Mar 2008, F7: 2PE. } \\
\text { M: } 25 \text { Feb 2007, F8: 2PE, det. P. Langton. }\end{array}$ \\
\hline Corynoneura celtica & $\mathrm{B}$ & B: 14 May 2007, F26: 1PE. \\
\hline Corynoneura gratias & $\mathrm{B}$ & B: 24 Sep 2007, S16: 3PE, det. P. Langton. \\
\hline Corynoneura lacustris & $\mathrm{CZ}(\mathrm{B}, \mathrm{M})$ & $\begin{array}{l}\text { B: } 29 \text { Apr 2008, F13: 1PE; } 24 \text { Sep 2007, F20: 6PE, } \\
\text { det. P. Langton; 09 Apr 2007, F30: 3PE, det. P. } \\
\text { Langton. } \\
\text { M: } 19 \text { Nov 2006, F15: 1PE, det. P. Langton; } 29 \text { Aug } \\
\text { 2006, F27: 1PE, det. P. Langton. }\end{array}$ \\
\hline Corynoneura lobata & $\mathrm{CZ}(\mathrm{B})$ & $\begin{array}{l}\text { B: } 29 \text { Apr 2008, F13: 7PE; } 24 \text { Sep 2007, F19: 7PE; } \\
\text { 09 Apr 2007, F22: 6PE, det. P. Langton. }\end{array}$ \\
\hline Corynoneura scutellata & M & $\begin{array}{l}\text { M: } 30 \text { Aug 2006, S9: 4PE; } 27 \text { Aug 2008, S20: 10PE, } \\
\text { leg. P. Komzák \& S. Větř́iček; } 28 \text { May 2008, S20: } \\
\text { 3PE, leg. P. Komzák \& S. Větř́čck. }\end{array}$ \\
\hline Cricotopus albiforceps & $\mathrm{B}$ & $\begin{array}{l}\text { B: } 29 \text { Apr 2008, F13: 4PE; } 24 \text { Apr 2007, F28: 1PE, } \\
\text { leg. M. Straka, all det. P. Langton. }\end{array}$ \\
\hline Cricotopus festivellus & $\mathrm{CZ}(\mathrm{B})$ & B: 23 Sep 2007, S19: 6PE, det. P. Langton. \\
\hline Cricotopus tremulus & $\mathrm{CZ}(\mathrm{B}, \mathrm{M})$ & $\begin{array}{l}\text { B: } 24 \text { Sep 2007, F20: 1PE, det. P. Langton. } \\
\text { M: } 29 \text { Apr 2007, F5: 4PE; } 04 \text { Oct 2002, F25: 1PE. }\end{array}$ \\
\hline Eukiefferiella coerulescens & B & $\begin{array}{l}\text { B: } 24 \text { Sep 2007, F19: 5PE; } 14 \text { May 2007, F26: 4PE, } \\
\text { det. P. Langton; } 23 \text { Sep 2007, F29: 6PE. }\end{array}$ \\
\hline Eukiefferiella devonica & M & $\begin{array}{l}\text { M: } 31 \text { Mar 2007, F2: 8PE; } 31 \text { Mar 2007, F3: 4PE; } 31 \\
\text { Mar 2007, F4: 7PE; } 31 \text { Mar 2007, F12: 6PE; } 03 \text { Oct } \\
\text { 2002, F25: 3PE. }\end{array}$ \\
\hline Eukiefferiella dittmari & $\mathrm{CZ}(\mathrm{B}, \mathrm{M})$ & $\begin{array}{l}\text { B: } 23 \text { Sep 2007, F29: 1PE, det. P. Langton. } \\
\text { M: } 31 \text { Mar 2007, F2: 1PE. }\end{array}$ \\
\hline Eukiefferiella fittkaui & $\mathrm{CZ}(\mathrm{M})$ & M: 18 May 2011, F9: 1PE; 20 Apr 2011, F9: 3PE. \\
\hline Eukiefferiella fuldensis & $\mathrm{B}$ & $\begin{array}{l}\text { B: } 20 \text { Apr 2007, F11: 10PE, det. P. Langton; } 24 \text { Sep } \\
\text { 2007, F19: 1PE; } 24 \text { Sep 2007, F20: 1PE; } 14 \text { May } \\
\text { 2007, F26: 5PE. }\end{array}$ \\
\hline Eukiefferiella tirolensis & $\mathrm{CZ}(\mathrm{M})$ & $\begin{array}{l}\text { M: } 31 \text { Mar 2007, F12: 1PE, det. P. Langton; } 03 \text { Oct } \\
\text { 2002, F25: 1PE; } 29 \text { Oct 2008, F25: 1PE. }\end{array}$ \\
\hline Heleniella ornaticollis & B & $\begin{array}{l}\text { B: } 20 \text { Apr 2007, F11: 2PE, det. P. Langton; } 14 \text { May } \\
\text { 2007, F26: 1PE. }\end{array}$ \\
\hline Heterotanytarsus apicalis & M & $\begin{array}{l}\text { M: } 09 \text { May 2006, } H 7: \text { 1P; } 25 \text { Sep 2006, } H 7: 6 \mathrm{P}+25 \mathrm{~L} \text {, } \\
\text { all leg. J. Bojková. }\end{array}$ \\
\hline
\end{tabular}




\begin{tabular}{|c|c|c|}
\hline Species & New to & Record details \\
\hline Hydrobaenus lugubris & M & M: 19 Nov 2006, S8: 1PE, det. P. Langton. \\
\hline Krenosmittia boreoalpina & $\mathrm{CZ}(\mathrm{B}, \mathrm{M})$ & $\begin{array}{l}\text { B: } 14 \text { May 2007, F26: 3PE. } \\
\text { M: } 18 \text { May 2011, F9: 2PE. }\end{array}$ \\
\hline Limnophyes edwardsi & $\mathrm{CZ}(\mathrm{B}, \mathrm{M})$ & $\begin{array}{l}\text { B: } 09 \text { Apr 2007, F22: 1PE, det. P. Langton. } \\
\text { M: } 31 \text { Mar 2007, F3: 1PE, det. P. Langton. }\end{array}$ \\
\hline Limnophyes punctipennis & $\mathrm{CZ}(\mathrm{M})$ & $\begin{array}{l}\text { M: } 21 \text { Apr 2008, S12: 1PE, leg. P. Komzák } \\
\text { \& S. Větř́iček; } 24 \text { Sep 2015, O1: } 1 \text { reared I } q \\
\text { (parthenogenetic) + } 2 \text { P, leg. K. Benesch. }\end{array}$ \\
\hline Limnophyes spinigus & $\mathrm{CZ}(\mathrm{B})$ & B: 23 Sep 2007, F29: 1PE, det. P. Langton. \\
\hline Nanocladius balticus & $\mathrm{CZ}(\mathrm{M})$ & $\begin{array}{l}\text { M: } 22 \text { Jul 2008, S20: 1PE; } 25 \text { Jun 2008, S20: 1PE; } \\
27 \text { Aug 2008, S20: 3PE, all leg. P. Komzák \& S. } \\
\text { Větříček. }\end{array}$ \\
\hline Nanocladius parvulus & $\mathrm{CZ}(\mathrm{B})$ & B: 14 May 2007, F26: 1PE. \\
\hline Orthocladius ashei & B & B: 24 Apr 2007, F28: 1PE, leg. M. Straka. \\
\hline Orthocladius glabripennis & M & $\begin{array}{l}\text { M: } 31 \text { Mar 2007, F2: 2PE, det. P. Langton; } 31 \mathrm{Mar} \\
\text { 2007, F3: 1PE; } 31 \text { Mar 2007, F4: 2PE; } 10 \text { Apr 2012, } \\
\text { F24: } 1 \text { reared pharate imago; } 21 \text { Apr 2008, S10: 1PE, } \\
\text { leg. P. Komzák \& S. Větř́čck; } 21 \text { Apr 2008, S11: 1PE, } \\
\text { leg. P. Komzák \& S. Větř́čck } 21 \text { Apr 2008, S12: 2PE, } \\
\text { leg. P. Komzák \& S. Větrríček. }\end{array}$ \\
\hline Orthocladius lignicola & $\mathrm{CZ}(\mathrm{B}, \mathrm{M})$ & $\begin{array}{l}\text { B: } 09 \text { Apr 2007, F30: 1PE. } \\
\text { M: } 29 \text { Apr 2007, H4: 1L; } 26 \text { Apr 2012, F10: 6L; } 18 \\
\text { May 2011, F9: 1PE; } 20 \text { Apr 2011, F9: 8PE. }\end{array}$ \\
\hline Orthocladius oblidens & B & B: 29 Apr 2008, F13: 1PE. \\
\hline Orthocladius pedestris & B & $\begin{array}{l}\text { B: } 20 \text { Apr 2007, F11: 5PE, det. P. Langton; } 29 \text { Apr } \\
\text { 2008, F13: 1PE. }\end{array}$ \\
\hline Orthocladius saxosus & B & B: 20 Apr 2007, F11: 5PE; 24 Sep 2007, F19: 4PE. \\
\hline Paracladius conversus & $\mathrm{CZ}(\mathrm{B})$ & $\begin{array}{l}\text { B: } 17 \text { Oct 2013, S7: 17PE; } 24 \text { Jul 2013, S7: 1PE; } 29 \\
\text { Aug 2013, S7: 1PE, all leg. D. Němejcová. }\end{array}$ \\
\hline Parakiefferiella bathophila & $\mathrm{CZ}(\mathrm{B}, \mathrm{M})$ & $\begin{array}{l}\text { B: } 29 \text { May 2015, S1: 6PE; } 30 \text { May 2015, S6: 1PE, leg. } \\
\text { D. Němejcová; } 28 \text { May 2015, S13: 10PE. M: } 03 \text { Oct } \\
\text { 2002, F25: 1PE, det. P. Langton; } 29 \text { Aug 2006, F27: } \\
\text { 1PE, det. P. Langton. }\end{array}$ \\
\hline Psectrocladius limbatellus & $\mathrm{B}$ & B: 23 Sep 2007, S19: 2PE. \\
\hline Psectrocladius octomaculatus & $\mathrm{CZ}(\mathrm{B})$ & B: 28 May 2015, S13: 1PE. \\
\hline Psectrocladius oxyura & $\mathrm{CZ}(\mathrm{B}, \mathrm{M})$ & $\begin{array}{l}\text { B: } 30 \text { May 2015, S6: 2PE; } 17 \text { Oct 2013, S7: 3PE; } 24 \\
\text { Jul 2013, S7: 1PE, all leg. D. Němejcová. } \\
\text { M: } 28 \text { Aug 2008, S10: 1PE; } 22 \text { Apr 2008, S20: 6PE; } \\
\text { 22 Jul 2008, S20: 2PE; } 25 \text { Jun 2008, S20: 2PE; } 27 \\
\text { Aug 2008, S20: 2PE; } 28 \text { May 2008, S20: 14PE, all } \\
\text { leg. P. Komzák \& S. Větŕíćek. }\end{array}$ \\
\hline Psectrocladius platypus & $\mathrm{CZ}(\mathrm{B})$ & B: 24 Sep 2007, S16: 11PE; 12 Oct 2008, S17: 1PE. \\
\hline Psectrocladius schlienzi & $\mathrm{CZ}(\mathrm{B})$ & B: 23 Sep 2007, S19: 4PE. \\
\hline Rheocricotopus atripes & $\mathrm{CZ}(\mathrm{M})$ & $\begin{array}{l}\text { M: } 11 \text { May 2006, H1: 1PE, leg. J. Bojková, det. P. } \\
\text { Langton. }\end{array}$ \\
\hline Rheocricotopus effusus & B & $\begin{array}{l}\text { B: } 24 \text { Sep 2007, F19: 5PE, det. P. Langton; } 14 \text { May } \\
\text { 2007, F26: 1PE. }\end{array}$ \\
\hline Thienemanniella vittata & B & B: 29 Apr 2008, F13: 3PE; 24 Sep 2007, F20: 1PE. \\
\hline
\end{tabular}




\begin{tabular}{|c|c|c|}
\hline Species & New to & Record details \\
\hline \multicolumn{3}{|l|}{ CHIRONOMINAE-Chironomini } \\
\hline Chironomus acidophilus & $\mathrm{CZ}(\mathrm{B})$ & B: 23 Apr 2007, S21: 1PE, det. P. Langton. \\
\hline Chironomus anthracinus & $\mathrm{CZ}(\mathrm{B})$ & B: 16 May 2007, S4: 2PE, det. P. Langton. \\
\hline Chironomus entis & M & M: 02 May 2005, F1: 1PE, det. P. Langton. \\
\hline Chironomus lacunarius & $\mathrm{CZ}(\mathrm{B})$ & $\begin{array}{l}\text { B: } 16 \text { May 2007, S4: 2PE; } 23 \text { Apr 2007, S21: 1PE, all } \\
\text { det. P. Langton. }\end{array}$ \\
\hline Chironomus lugubris & $\mathrm{CZ}(\mathrm{B})$ & B: 20 Aug 2006, S18: 1PE, det, P. Langton. \\
\hline Chironomus luridus & M & $\begin{array}{l}\text { M: } 02 \text { Sep 2006, O2: 4PE, leg. J. Sychra, det. P. } \\
\text { Langton. }\end{array}$ \\
\hline Chironomus montuosus & $\mathrm{CZ}(\mathrm{B})$ & B: 16 May 2007, S4: 6PE, det. P. Langton. \\
\hline Cladopelma virescens & $\mathrm{B}$ & B: 30 Aug 2013, S3: 2PE, leg. D. Němejcová. \\
\hline Cladopelma viridulum & $\mathrm{CZ}(\mathrm{B})$ & B: 30 Aug 2013, S3: 2PE, leg. D. Němejcová. \\
\hline Cryptochironomus obreptans & B & B: 29 Aug 2013, S7: 1PE, leg. D. Němejcová. \\
\hline Cryptochironomus supplicans & $\mathrm{CZ}(\mathrm{M})$ & $\begin{array}{l}\text { M: } 22 \text { Jul 2008, S20: 3PE; } 25 \text { Jun 2008, S20: 1PE, all } \\
\text { leg. P. Komzák \& S. Větrríček. }\end{array}$ \\
\hline Cryptotendipes holsatus & $\mathrm{CZ}(\mathrm{M})$ & M: 30 Aug 2006, S9: 5PE. \\
\hline Cryptotendipes pseudotener & M & M: 31 Jul 2012, F14: 1 reared P. \\
\hline Cryptotendipes usmaensis & $\mathrm{CZ}(\mathrm{B}, \mathrm{M})$ & $\begin{array}{l}\text { B: } 24 \text { Jul 2013, S3: 2PE; } 24 \text { Jul 2013, S7: 9PE, } \\
\text { leg. D. Němejcová; } 29 \text { Aug 2013, S7: 1PE, leg. D. } \\
\text { Němejcová. } \\
\text { M: } 24 \text { Jun 2008, S11: 1PE, leg. P. Komzák \& S. } \\
\text { Větř́ček; } 28 \text { Aug 2008, S11: 1PE, leg. P. Komzák \& } \\
\text { S. Větříček. }\end{array}$ \\
\hline Dicrotendipes pulsus & $\mathrm{CZ}(\mathrm{M})$ & $\begin{array}{l}\text { M: } 22 \text { Jul 2008, S20: 70PE, leg. P. Komzák \& S. } \\
\text { Větříček; } 25 \text { Jun 2008, S20: 39PE, leg. P. Komzák \& } \\
\text { S. Větříček; } 27 \text { Aug 2008, S20: 25PE, leg. P. Komzák } \\
\text { \& S. Větříček. }\end{array}$ \\
\hline Glyptotendipes paripes & M & $\begin{array}{l}\text { M: } 24 \text { Jun 2008, S10: 6PE; } 28 \text { Jul 2008, S10: 1PE; } 29 \\
\text { May 2008, S10: 1PE; } 24 \text { Jun 2008, S11: 25PE; } 28 \text { Jul } \\
\text { 2008, S11: 4PE; 24 Jun 2008, S12: 4PE; 28 Aug 2008, } \\
\text { S12: 1PE; } 28 \text { Jul 2008, S12: 14PE; 29 May 2008, S12: } \\
\text { 3PE, all leg. P. Komzák \& S. Větř́čck. }\end{array}$ \\
\hline Harnischia curtilamellata & $\mathrm{CZ}(\mathrm{B}, \mathrm{M})$ & $\begin{array}{l}\text { B: } 24 \text { Jul 2013, S3: 1PE; } 24 \text { Jul 2013, S7: 3PE, } \\
\text { leg. D. Němejcová; } 29 \text { Aug 2013, S7: 1PE, leg. D. } \\
\text { Němejcová. } \\
\text { M: } 24 \text { Jun 2008, S11: 1PE, leg. P. Komzák \& S. } \\
\text { Větříček. }\end{array}$ \\
\hline Kiefferulus tendipediformis & B & B: 30 Aug 2013, S3: 2PE, leg. D. Němejcová. \\
\hline Microchironomus tener & B & $\begin{array}{l}\text { B: } 23 \text { Jul 2013, S5: 1PE; } 29 \text { Aug 2013, S5: 1PE, all } \\
\text { leg. D. Němejcová. }\end{array}$ \\
\hline Microtendipes confinis & $\mathrm{CZ}(\mathrm{B})$ & $\begin{array}{l}\text { B: } 24 \text { Apr 2007, F28: 1PE, leg. M. Straka, det. P. } \\
\text { Langton. }\end{array}$ \\
\hline Microtendipes diffinis & B & B: 29 Apr 2008, F13: 3PE, det. P. Langton. \\
\hline Paracladopelma nigritula & $\mathrm{CZ}(\mathrm{B})$ & B: 24 Jul 2013, S7: 37PE, leg. D. Němejcová. \\
\hline Parachironomus parilis & B & B: 23 Sep 2007, S19: 1PE. \\
\hline Parachironomus vitiosus & $\mathrm{CZ}(\mathrm{M})$ & $\begin{array}{l}\text { M: } 28 \text { May 2008, S20: 2PE, leg. P. Komzák \& S. } \\
\text { Větríiček. }\end{array}$ \\
\hline Paralauterborniella nigrohalteralis & $\mathrm{CZ}(\mathrm{M})$ & M: 25 Jul 2012, F17: 1PE. \\
\hline
\end{tabular}




\begin{tabular}{|c|c|c|}
\hline Species & New to & Record details \\
\hline Paratendipes nudisquama & $\mathrm{CZ}(\mathrm{M})$ & $\begin{array}{l}\text { M: } 10 \text { Jul 2006, H7: 3L; } 29 \text { Apr 2007, H9: 31L, all } \\
\text { leg. J Bojková. }\end{array}$ \\
\hline Phaenopsectra flavipes & $\mathrm{B}$ & $\begin{array}{l}\text { B: } 29 \text { May 2015, S1: 20PE; } 29 \text { May 2015, S2: 3PE; } 30 \\
\text { Aug 2013, S3: 3PE, leg. D. Němejcová; } 28 \text { May 2015, } \\
\text { S13: 20PE; } 30 \text { May 2015, S14: 9PE. }\end{array}$ \\
\hline Polypedilum albicorne & $\mathrm{CZ}(\mathrm{B}, \mathrm{M})$ & $\begin{array}{l}\text { B: } 24 \text { Sep } 2007, F 20: 1 \text { PE, det. P. Langton; } 29 \text { May } \\
\text { 2015, S2: 1PE; } 28 \text { May 2015, S13: 8PE. } \\
\text { M: } 03 \text { Oct 2002, F25: 1PE; } 29 \text { Aug 2006, F27: 1PE. }\end{array}$ \\
\hline Polypedilum nubens & $\mathrm{CZ}(\mathrm{M})$ & $\begin{array}{l}\text { M: } 25 \text { Sep 2008, S12: 1PE; } 22 \text { Jul 2008, S20: 2PE; } \\
27 \text { Aug 2008, S20: 16PE, all leg. P. Komzák \& S. } \\
\text { Větríček. }\end{array}$ \\
\hline Polypedilum sordens & $\mathrm{CZ}(\mathrm{B}, \mathrm{M})$ & $\begin{array}{l}\text { B: } 24 \text { Jul 2013, S3: 1PE; } 23 \text { Jul 2013, S5: 2PE, leg. D. } \\
\text { Němejcová. } \\
\text { M: } 25 \text { Sep 2008, S11: 5PE, leg. P. Komzák \& S. } \\
\text { Větř́ček; } 28 \text { Jul 2008, S12: 2PE, leg. P. Komzák \& S. } \\
\text { Větríčck. }\end{array}$ \\
\hline Polypedilum tritum & $\mathrm{CZ}(\mathrm{M})$ & $\begin{array}{l}\text { M: } 25 \text { Jun 2008, S20: 1PE, leg. P. Komzák \& S. } \\
\text { Větř́íck. }\end{array}$ \\
\hline Robackia demeijerei & $\mathrm{CZ}(\mathrm{M})$ & M: $25 \mathrm{Jul}$ 2012, F17: 1L. \\
\hline Stenochironomus gibbus & $\mathrm{CZ}(\mathrm{B})$ & B: 24 Jul 2013, S7: 1PE, leg. D. Němejcová. \\
\hline Tribelos intextum & $\mathrm{CZ}(\mathrm{B})$ & B: 29 May 2015, S1: 14PE. \\
\hline Tribelos intextus & $\mathrm{CZ}(\mathrm{B})$ & B: 24 Jul 2013, S3: 1PE. \\
\hline Xenochironomus xenolabis & M & $\begin{array}{l}\text { M: } 24 \text { Jun 2008, S10: 1PE; } 28 \text { Aug 2008, S10: 2PE; } \\
29 \text { May 2008, S10: 2PE, all leg. P. Komzák \& S. } \\
\text { Větríčck. }\end{array}$ \\
\hline \multicolumn{3}{|l|}{ CHIRONOMINAE-Tanytarsini } \\
\hline Cladotanytarsus lepidocalcar & $\mathrm{CZ}(\mathrm{M})$ & $\begin{array}{l}\text { M: } 30 \text { Aug 2006, S9: 1PE, det. P. Langton; } 28 \text { Jul } \\
\text { 2008, S10: 4PE, leg. P. Komzák \& S. Větř́čček; } 25 \\
\text { Sep 2008, S11: 7PE, leg. P. Komzák \& S. Větrríček; } 28 \\
\text { Aug 2008, S11: 9PE, leg. P. Komzák \& S. Větříček; } \\
28 \text { Aug 2008, S12: 3PE, leg. P. Komzák \& S. } \\
\text { Větríček; 28 Jul 2008, S12: 2PE, leg. P. Komzák \& S. } \\
\text { Větříček; 22 Jul 2008, S20: 59PE, leg. P. Komzák \& } \\
\text { S. Větříček; 25 Jun 2008, S20: 31PE, leg. P. Komzák } \\
\text { \& S. Větríiček; 27 Aug 2008, S20: 104PE, leg. P. } \\
\text { Komzák \& S. Větříček; 28 May 2008, S20: 8PE, leg. } \\
\text { P. Komzák \& S. Větříček. }\end{array}$ \\
\hline Lithotanytarsus emarginatus & $\begin{array}{l}\mathrm{CZ}(\mathrm{M}) \\
\mathrm{SK}\end{array}$ & $\begin{array}{l}\text { M: } 20 \text { Apr 2011, F9: 4L. } \\
\text { SK: } 13 \text { May 2012, F6: 5L; } 22 \text { Apr 2013, F31: 2L. }\end{array}$ \\
\hline Micropsectra apposita & $\mathrm{CZ}(\mathrm{M})$ & M: 31 Mar 2007, F4: 4PE, det. P. Langton. \\
\hline Micropsectra atrofasciata & $\mathrm{CZ}(\mathrm{B}, \mathrm{M})$ & $\begin{array}{l}\text { B: } 24 \text { Sep 2007, F19: 5PE; } 24 \text { Sep 2007, F20: 12PE; } \\
\text { 09 Apr 2007, F22: 4PE, det. P. Langton. } \\
\text { M: } 31 \text { Mar 2007, F2: 4PE, det. P. Langton; } 31 \text { Mar } \\
\text { 2007, F4: 7PE; } 14 \text { Oct 2007, F23: 2PE; 29 Oct 2008, } \\
\text { F25: 4PE. }\end{array}$ \\
\hline Micropsectra bidentata & B & B: 29 Apr 2008, F13: 1PE; 24 Sep 2007, F19: 3PE. \\
\hline Micropsectra junci & $\mathrm{B}$ & B: 24 Sep 2007, F20: 1PE, det. P. Langton. \\
\hline Micropsectra lindrothi & $\mathrm{CZ}(\mathrm{B}, \mathrm{M})$ & $\begin{array}{l}\text { B: } 09 \text { Apr 2007, F22: } 1 \text { PE. } \\
\text { M: } 30 \text { Aug 2006, S9: 1PE, all det. P. Langton. }\end{array}$ \\
\hline
\end{tabular}




\begin{tabular}{|c|c|c|}
\hline Species & New to & Record details \\
\hline Micropsectra longicrista & $\mathrm{CZ}(\mathrm{M})$ & M: 04 May 2009, H6: 2I ${ }^{\lambda}$. \\
\hline Micropsectra pallidula & $\begin{array}{l}\mathrm{CZ}(\mathrm{M}) \\
\text { SK }\end{array}$ & $\begin{array}{l}\text { M: } 14 \text { Sep 2011, H8: 1P. } \\
\text { SK: } 13 \text { Sep 2011, H2: 1P. }\end{array}$ \\
\hline Neostempellina thienemanni & $\mathrm{CZ}(\mathrm{M})$ & $\begin{array}{l}\text { M: } 29 \text { Apr 2007, H4: 1P+5L; } 29 \text { Apr 2007, H10: } \\
\text { 1P+3L, all leg. J. Bojková. }\end{array}$ \\
\hline Paratanytarsus bituberculatus & B & B: 24 Jul 2013, S7: 1PE, leg. D. Němejcová. \\
\hline Paratanytarsus penicillatus & $\mathrm{CZ}(\mathrm{B})$ & B: 23 Apr 2007, S21: 1PE, det. P. Langton. \\
\hline Rheotanytarsus muscicola & M & $\begin{array}{l}\text { M: } 02 \text { May 2005, F1: 1PE, det. P. Langton; } 03 \text { Oct } \\
\text { 2002, F25: 1PE. }\end{array}$ \\
\hline Stempellina almi & $\mathrm{CZ}(\mathrm{B}, \mathrm{M})$ & $\begin{array}{l}\text { B: } 24 \text { Jul 2013, S7: 7PE, leg. D. Němejcová. } \\
\text { M: } 27 \text { Aug 2008, S20: 1PE, leg. P. Komzák \& S. } \\
\text { Větříček. }\end{array}$ \\
\hline Stempellinella edwardsi & $\mathrm{CZ}(\mathrm{B}, \mathrm{M})$ & $\begin{array}{l}\text { B: } 29 \text { Apr 2008, F13: 18PE; } 24 \text { Apr 2007, F28: 3PE, } \\
\text { leg. M. Straka. } \\
\text { M: } 22 \text { Jul 2008, S20: 3PE, leg. P. Komzák \& S. } \\
\text { Větříček; } 25 \text { Jun 2008, S20: 2PE, leg. P. Komzák \& S. } \\
\text { Větříček; } 27 \text { Aug 2008, S20: 46PE, leg. P. Komzák \& } \\
\text { S. Větříček. }\end{array}$ \\
\hline Stempellinella flavidula & $\mathrm{CZ}(\mathrm{M})$ & $\begin{array}{l}\text { M: } 29 \text { Apr 2007, H4: 8PE, leg. J. Bojková; } 18 \text { May } \\
\text { 2011, F9: 2PE; } 31 \text { Aug 2006, F18: 1PE, det. P. } \\
\text { Langton. }\end{array}$ \\
\hline Tanytarsus bathophilus & $\mathrm{CZ}(\mathrm{B}, \mathrm{M})$ & $\begin{array}{l}\text { B: } 24 \text { Jul 2013, S7: 21PE, leg. D. Němejcová. } \\
\text { M: } 22 \text { Jul 2008, S20: 41PE; } 25 \text { Jun 2008, S20: 12PE; } \\
27 \text { Aug 2008, S20: 4PE; } 28 \text { May 2008, S20: 8PE, all } \\
\text { leg. P. Komzák \& S. Větrríček. }\end{array}$ \\
\hline Tanytarsus curticornis & $\mathrm{CZ}(\mathrm{M})$ & $\begin{array}{l}\text { M: } 02 \text { May 2005, F1: 2PE, det. P. Langton; } 28 \text { Aug } \\
\text { 2008, S11: 1PE, leg. P. Komzák \& S. Větríćck. }\end{array}$ \\
\hline Tanytarsus debilis & $\mathrm{CZ}(\mathrm{B})$ & B: 30 May 2015, S14: 1PE. \\
\hline Tanytarsus ejuncidus & M & $\begin{array}{l}\text { M: } 24 \text { Jun 2008, S10: } 2 \text { PE; } 28 \text { Aug 2008, S11: 2PE; } \\
22 \text { Jul 2008, S20: 2PE; } 27 \text { Aug 2008, S20: 14PE, all } \\
\text { leg. P. Komzák \& S. Větŕíček. }\end{array}$ \\
\hline Tanytarsus eminulus & M & M: 19 Nov 2006, F15: 2PE, det. P. Langton. \\
\hline Tanytarsus longitarsis & $\mathrm{CZ}(\mathrm{B})$ & B: 29 Apr 2008, F13: 29PE, det. P. Langton. \\
\hline Tanytarsus medius & $\mathrm{CZ}(\mathrm{B}, \mathrm{M})$ & $\begin{array}{l}\text { B: } 24 \text { Jul 2013, S7: 1PE, leg. D. Němejcová. } \\
\text { M: } 29 \text { May 2008, S10: 9PE, leg. P. Komzák \& S. } \\
\text { Větř́icek. }\end{array}$ \\
\hline Tanytarsus mendax & M & $\begin{array}{l}\text { M: } 30 \text { Aug 2006, S9: 1PE, det. P. Langton; } 24 \text { Jun } \\
\text { 2008, S10: 1PE, leg. P. Komzák \& S. Větríček. }\end{array}$ \\
\hline Tanytarsus telmaticus & $\mathrm{CZ}(\mathrm{M})$ & M: 19 Nov 2006, S8: 1PE, det. P. Langton. \\
\hline
\end{tabular}




\section{References}

Ashe, P., Moubayed-Breil, J. and Vondrák, D. 2014. First records of Buchonomyia thienemanni Fittkau (Diptera: Chironomidae) from the Czech Republic. - CHIRONOMUS Newsletter on Chironomidae Research 27: 51-53.

Bitušík, P. and Brabec, K. 2009. Chironomidae Newman, 1834. In: Jedlička, L., Kúdela, M. and Stloukalová, V. (eds). Checklist of Diptera of the Czech Republic and Slovakia. Electronic version 2. http:// zoology.fns.uniba.sk/diptera2009

Brundin, L. 1966. Transantarctic relationships and their significance, as evidenced by chironomid midges. With a monograph on the subfamilies Podonominae and Aphroteniinae and the austral Heptagyiae. Kunglica Svenska Vetenskapsakademiens Handlingar 11: 1-472.

Lencioni, V., Marziali, L. and Rossaro, B. 2007. The first record of Parochlus kiefferi (Garrett, 1925) (Diptera, Chironomidae, Podonominae) from Italy. - Entomological News 118: 127-133.

Sæther, O.A. and Spies, M. 2015. Fauna Europaea: Family Chironomidae. Fauna Europaea version 2.6.2, http://www.faunaeur.org

Skála, I. 2011. Faunistic records from the Czech Republic - 318. Diptera: Chironomidae. - Klapalekiana 47: 265-269.

Skála, I. 2012. Faunistic records from the Czech Republic - 331. Diptera: Chironomidae. - Klapalekiana 48: 151-156.

Skála, I. 2013. Faunistic records from the Czech Republic - 347. Diptera: Chironomidae. - Klapalekiana 49: 107-108.

Skála, I. 2014. Faunistic records from the Czech Republic - 372. Diptera: Chironomidae. - Klapalekiana 50: $257-258$.

Verberk, W.C.E.P., Van Duinen, G.A., Moller Pillot, H.K.M. and Esselink, H. 2003. Lasiodiamesa gracilis (Chironomidae: Podonominae) new for the Dutch fauna. - Entomologische Berichten 63: 40-42.

Wülker, W. 1958. Die Bedeutung der Chironomiden für die limnologisch-tiergeographische Charakterisierung der Hochschwarzwaldes. - Verhandlugen der Internationale Vereinigung für Theoretische und Angewandte Limnologie 13: 805-813. 
Appendix 1. Collection sites with details on their location and protection status.

\section{Helocrene springs}

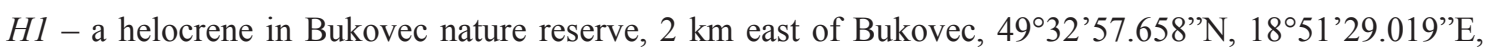
Moravia, CZ.

$H 2$ - a helocrene in the floodplain of river Hron, south bank, approx. $2 \mathrm{~km}$ upstream Červená Skala, $48^{\circ} 49^{\prime} 35.645^{\prime \prime} \mathrm{N}, 20^{\circ} 9^{\prime} 47.527^{\prime \prime} \mathrm{E}$, Slovakia.

$H 3$ - a helocrene on the south margin of Horní Lomná 49³1'6.288”, 18³7’48.932”, CZ, Moravia.

$H 4$ - helocrene Kalábová, nature monument, 1.5 km north of Březová, $48^{\circ} 56^{\prime} 23.788^{\prime}{ }^{\prime} \mathrm{N}, 17^{\circ} 44^{\prime} 38.353^{\prime \prime} \mathrm{E}$, Moravia, CZ.

H5 - helocrene, Kyčmol nature monument, $2 \mathrm{~km}$ southwest of Horní Lomná, 49³0'46.607’N, 18³7’24.557’'E, Moravia, CZ.

H6 - a helocrene approx. $1 \mathrm{~km}$ southeast of Lopeník 4856’33.653”N, 1747’52.779”E, CZ, Moravia.

$H 7$-a helocrene in Obidová nature monument, $400 \mathrm{~m}$ southwest of Visalaje $49^{\circ} 31^{\prime} 1.171$ 'N, 18 31 '26.765”'E, Moravia, $\mathrm{CZ}$.

$H 8$ - helocrene V Krátkých, nature monument, approx. 2 km southwest of Vápenice, 4857’24.443”N, 17²9’28.765’'E, Moravia, CZ.

H9-helocrene Chmelinec, nature monument, directly south of Vyškovec, 48 $56^{\prime} 25.587^{\prime \prime} \mathrm{N}, 17^{\circ} 51^{\prime} 19.175^{\prime \prime} \mathrm{E}$, Moravia, $\mathrm{CZ}$.

$H 10$ - a helocrene in Hutě nature reserve, approx. 2 km northeast of Žítková 4859’25.389’'N, 1754'19.785'E, Moravia, CZ.

$H 11$ - a helocrene spring above the road about 100 m east of Nová Klínovka chalet (formerly Tesla) in the eastern part of a meadow called Klínové Boudy. About 4 km southeast of Špindlerův Mlýn, The Krkonoše Mountains National Park, 5042’30.812”N, 15³9’35.048”E, about 1230 m a.s.l., Bohemia, CZ. Fig. 2.

\section{Flowing waters}

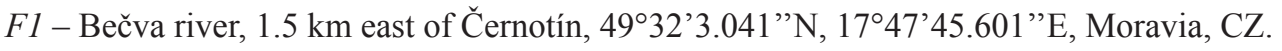

F2 - Bobrůvka (brook), 700 m upstream of Olešínky, 49²9'9.02”N, 16²'53.64”E, Moravia, CZ.

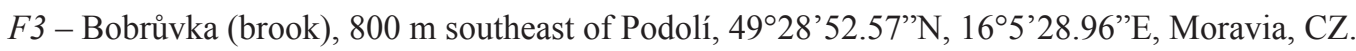

F4 - Bobrůvka (brook) 1 km north of Řečice, 49³1'25.29’'N, 16³'49.59’'E, Moravia, CZ.

F5 - Bobrůvka (brook) $400 \mathrm{~m}$ upstream of Strážek, 49²6'42.52”N, 16¹1'21.45”E, Moravia, CZ.

F6 - Čierný potok (brook), 1.5 km west od Súl'ov, 499'44.064”'N, 18³4’4.584”'E, Slovakia.

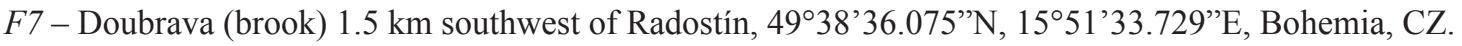

F8 - Fryšávka (brook) 500 m upstream of Jimramov, 49³8'12.293”N, 16²12'59.322”E, Moravia, CZ.

F9 - a brook flowing from south and touching Chmelinec nature monument at its eastern margin, $160 \mathrm{~m}$ east of Vyškovec 48 $56^{\prime} 26.357^{\prime} ’ \mathrm{~N}, 17^{\circ} 51^{\prime} 19.527^{\prime} \mathrm{E}$, Moravia, CZ.

F10 - Jasénka brook, $3 \mathrm{~km}$ upstream (northeast) of Horní Jasénka, 49²2’36.487’'N, 18¹'26.930”E, Moravia, CZ.

F11 - Křemelná (brook), 3 km downstream of Prášily, 498’7.17’”, 1323’7.76”E, Bohemia, CZ.

F12 - Bobrůvka (brook), $1 \mathrm{~km}$ east of Mirošov, above the bridge, 49 $27^{\prime} 56.47^{\prime \prime} \mathrm{N}, 16^{\circ} 10^{\prime} 25.28^{\prime \prime} \mathrm{E}$, Moravia, CZ.

F13 - Lužnice (river), Horní Lužnice nature reserve, approx. 2 km upstream of Halámky, 4850'7.093”N, 1455'41.59’'E, Bohemia, CZ.

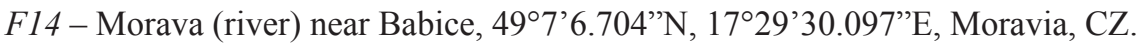


F15 - Morava (river) just at the confluence with Dyje (river), $12 \mathrm{~km}$ south of Lanžhot, 48³7’2.400”N, 1656'26.470”'E, Moravia, CZ.

F16 - Morava (river), near Spytihněv 49²'23.085'N, 17³0'14.110”E, Moravia, CZ.

F17 - Morava (river), Osypané Břehy nature monument, 3 km northwest of Strážnice, 4855'17.694”N, $17^{\circ} 16^{\prime} 44.529^{\prime \prime}$, Moravia, CZ.

F18 - Radějovka (brook), below the Kejda fish-pond, $5 \mathrm{~km}$ upstream of Radějov, 48 $52^{\prime} 3.635^{\prime \prime} \mathrm{N}$, 17²4'25.935'E, Moravia, CZ.

F19 - Roklanský potok (brook), Bohemian Forest NP, Modrava 1 km upstream of Rybárna, $3.5 \mathrm{~km}$ upstream of Modrava, 49¹'59.253”N, 1327'37.472”E, Bohemia, CZ.

F20 - Řezná (brook), Bohemian Forest NP, $1.5 \mathrm{~km}$ upstream of Železná Ruda, 498’47.614”N, $13^{\circ} 15^{\prime} 3.633^{\prime \prime} \mathrm{E}$, Bohemia, CZ.

F21 - a small brook on the northern margin of Senotín, 49 $4^{\prime} 11.328^{\prime \prime} \mathrm{N}, 15^{\circ} 8^{\prime} 35.034^{\prime \prime} \mathrm{E}$, Bohemia, CZ.

F22 - Sklenský potok (brook), Světnovské údolí nature monument, 1,5 km northeast of Světnov,

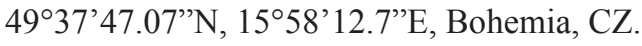

F23 - Svratka (brook), approx. 1.5 km north of Cikháj, 49³9’25.453”N, 1558'19.544”E, Moravia, CZ.

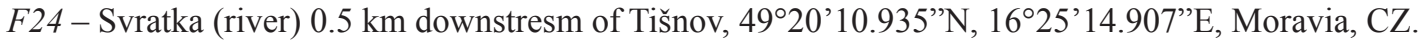

F25 - Svratka (river) 0.5 km downstream of Unčín, 49³6’48.839”N, 16¹4'4.124”E, Moravia, CZ.

F26 - Úhlava (river), just upstream of the Zadní Hamry bridge, approx. $2 \mathrm{~km}$ upstream of Hamry, $49^{\circ} 12^{\prime} 13.931$ 'N, $13^{\circ} 11^{\prime} 0.702^{\prime \prime} \mathrm{E}$, Bohemia, CZ.

F27 - Velička (brook), just upstream the downstream bridge in Vápenky, 48 52'29.032'N, 17³7'56.017’E, Moravia, CZ.

F28 - Vltava (river), upstream the bridge in Hluboká, 49²'58.664'N, 14²6’46.393”'E, Bohemia, CZ.

F29 - Vltava (river), Bohemian Forest NP, just downstream the bridge 1 km west of Pěkná, 4851'7.639'N, 1355'13.344"E, Bohemia, CZ.

F30 - Vortovský potok (brook), just downstream a bridge, $0.5 \mathrm{~km}$ upstream (south) Zlámanec fish-pond, $1.5 \mathrm{~km}$ upstream of Vortová, 4941'52.39'N, 1556'19.49”E, Bohemia, CZ.

F31 - a brook flowing from the Strážov hill to the southwest, approx $50 \mathrm{~m}$ upstream a bridge, 1.8 southeast

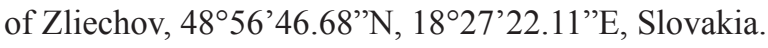

\section{Standing waters}

$S 1$ - Černé lake, Bohemian Forest NP, approx. 6 km northwest of Železná Ruda, 49¹0'53.643”N, $13^{\circ} 11^{\prime} 8.634$ 'E, Bohemia, CZ.

S2 - Čertovo lake, Bohemian Forest NP, approx. 4 km northwest of Železná Ruda, 499'54.959”'N, $13^{\circ} 12^{\prime} 0.216^{\prime \prime} \mathrm{E}$, Bohemia, CZ.

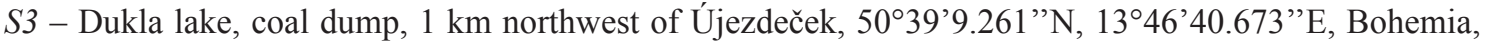
$\mathrm{CZ}$.

S4 - peat pool, Chalupská slat' raised bog nature monument, Bohemian Forest NP, approx. $1 \mathrm{~km}$ north of Borová Lada, 4859'53.44”N, 1339’33.65”E, Bohemia, CZ.

S5 - Kateřina lake, coal dump, $0.5 \mathrm{~km}$ south of Soběchleby, $50^{\circ} 39^{\prime} 51.5^{\prime \prime} \mathrm{N}, 13^{\circ} 53^{\prime 2} 21.9^{\prime \prime} \mathrm{E}$, Bohemia, CZ.

S6 - Laka lake, Bohemian Forest NP, 4 km south of Nová Hůrka, 496'36.771’N, 13¹9’37.522”E, Bohemia, $\mathrm{CZ}$.

$S 7$ - Lake ČSM, coal dump, $0.5 \mathrm{~km}$ southwest of Dubí-Pozorka, $50^{\circ} 39^{\prime} 18.5^{\prime \prime} \mathrm{N}, 13^{\circ} 46^{\prime} 54.9^{\prime \prime}$, Bohemia, CZ.

$S 8$ - an oxbow in the floodplain area of the confluence of Morava and Dyje rivers, approx. $10 \mathrm{~km}$ south of Lanžhot, 48³7’44.426”N, 1656’53.685’E, Moravia, CZ. 
$S 9$ - the downstream fish pond in Lopeník, 48 56'53.604”N, 1746’37.796”'E, Moravia, CZ.

S10 - Nové Mlýny - lower reservoir, just northeast of Pavlov 4853’2.104”'N, 16²41'11.455”E, Moravia, $\mathrm{CZ}$.

S11 - Nové Mlýny - middle reservoir, just northwest of Dolní Věstonice 4853’28.868’N, 16³8’18.291’”, Moravia, CZ.

S12 - Nové Mlýny - upper reservoir, south of Pasohlávky, 4853'58.676”N, 16³4'53.342”E, Moravia, CZ.

S13 - Plešné lake, Bohemian Forest NP, approx 4 km south of Jelení, 4846’39.051”N, 1352’4.220”E, Bohemia, CZ.

S14 - Prášilské lake, Bohemian Forest NP, approx $3.5 \mathrm{~km}$ southeast of Prášily, 494’31.638”N, 13²4'4.509'E, Bohemia, CZ.

S15 - a peat pool in Radostínské rašeliniště, mineral poor Sphagnum fen national nature reserve, $1 \mathrm{~km}$ northeast of Radostín, 49³9'23.37’N, 1553'14.82”E, Bohemia, CZ. Fig. 1.

S16 - a peat pool in Modravské slatě raised bog nature monument, Bohemian Forest NP, approx. $5.5 \mathrm{~km}$ west of Modrava, $49^{\circ} 1^{\prime} 26.588^{\prime}$ 'N, $13^{\circ} 25^{\prime} 0.427^{\prime}$ 'E, Bohemia, CZ.

$S 17$ - a peat pool in Rolavská vrchoviště raised bog national nature reserve, approx. $5 \mathrm{~km}$ north of Přebuz $50^{\circ} 24^{\prime} 32.387^{\prime \prime} \mathrm{N}, 12^{\circ} 36^{\prime} 33.625^{\prime \prime} \mathrm{E}$, Bohemia, CZ.

S18 - a pool on a small brook on the northern margin of Senotín, $49^{\circ} 4^{\prime} 11.328^{\prime \prime} \mathrm{N}, 15^{\circ} 8^{\prime} 35.034^{\prime \prime} \mathrm{E}$, Bohemia, $\mathrm{CZ}$.

S19 - an oxbow in the floodplain of Vltava (river), Bohemian Forest NP, north of the road from Pěkná to Pěkná railway station, $1.3 \mathrm{~km}$ west of Pěkná, 48 $51^{\prime} 6.654^{\prime \prime N}$, 1354'56.386”E, Bohemia, CZ.

S20 - Vranov reservoir, 0.5 km north of Vranov nad Dyjí, 4854'21.579’'N, 1549'4.072”'E, Moravia, CZ.

$S 21$ - a pool, Bohemian Forest NP, approx 700 m northeast of Pamferova Hut' and $3 \mathrm{~km}$ northeast of Železná Ruda, 499'15.772”N, 1315'46.462”'E, Bohemia, CZ.

\section{Other}

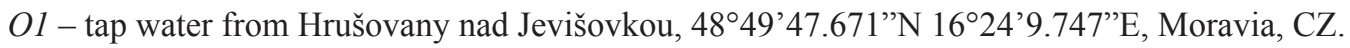

O2 - barrel with rain water, Rapotice, 49¹1'32.361'N, 16¹5'14.252”E, Moravia, CZ. 